\title{
Stabilité et émission $X$ d'un Z-pinch produit par un liner creux en aluminium
}

\author{
R. Rosch, D. Friart, M. Darrigol, L. Chatrieux, P. Zehnter, \\ P. Romary* et J.M. Chevalier* \\ CEA Bruyères-le-Châtel, BP. 12, 91680 Bruyères-le-Châtel, France \\ * CEA, CESTA, BP. 2, 33114 Le Barp, France
}

\begin{abstract}
Résumé : Des expériences précédentes à un niveau de puissance de $0,1 \mathrm{TW}$ [1] ont montré que la stabilité et le rayonnement $\mathrm{X}$ sont fortement ameliorés si on implose un liner en aluminium sur un fil central micrométrique. Nous présentons ici les résultats de l'implosion d'un jet d'aluminium à une puissance de 2 TW. Les expériences, réalisées sur le generrateur haute puissance pulsé AMBIORIX du CESTA sont largement instrumentées. Nous étudions l'effet du fil central et de sa nature sur la stabilité, l'homogénéité, la reproductibilité et le rendement $\mathrm{X}$. Les configurations de jet Al sur fil central en Al améliorent d'un facteur 2 la quantité d'énergie rayonné dans les raies $\mathrm{K}$ de l'aluminium $(1,5 \mathrm{keV}$ ou $7,7 \AA)$. L'interprétation à l'aide d'un modèle collisionnel-radiatif hors ETL, est en bon accord avec un plasma à quelques centaines d'eV et à quelques $10^{20} \mathrm{~cm}^{-3}$.
\end{abstract}

\section{INTRODUCTION}

Les Z-pinchs constituent des sources de rayonnement $X$ intenses et brèves [2]. Toutefois, l'apparition d'instabilités Rayleigh-Taylor limite le rendement de conversion de l'énergie en X. Les configurations à étage [1] permettent de réduire ces instabilités : on choisit ici de faire passer le courant dans une gaine cylindrique de vapeur d'aluminium (facilement ionisable), et de réaliser l'implosion de la gaine, seule ou sur un fil central en Al.

\section{DISPOSITIF EXPÉRIMENTAL}

Les expériences ont été réalisées sur l'installation AMBIORIX $(0,5 \Omega, 2 \mathrm{TW}, 2 \mathrm{MA}, 50 \mathrm{~ns})$. Le jet est formé par l'explosion d'une feuille de $3 \mu \mathrm{m}$ d'épaisseur à partir d'un banc de condensateurs externe (Fig. 1). L'interférométrie du jet a montré que l'essentiel de l'aluminium se trouve sous forme atomique, et remplit rapidement tout l'espace inter-électrodes. La projection du jet sur une plaque en graphite située au niveau de l'anode, a mis en évidence la présence d'une partie notable de la masse sous forme de gouttelettes (de 1 à $100 \mu \mathrm{m}$ de diamètre) [3].

Les diagnostics mis en oeuvre comprennent: une caméra à balayage de fente visible (phase hydrodynamique), deux spectromètres à cristal courbe en Mica et RbAp à haute résolution spectrale $(1 \mathrm{eV})$ pour évaluer l'énergie contenue dans les raies $\mathrm{K}$ (phase radiative); un trichromateur à trois cristaux plans en KAP pour le suivi temporel de trois raies principales de la couche $K$; un système d'imagerie à galette de microcanaux pulsée, donne des instantanés du plasma avec une résolution temporelle (1 ns) et spatiale $(300 \mu \mathrm{m})$; un ensemble de détecteurs photoélectriques et photoconducteurs permet de reconstruire le spectre d'émission du plasma par la méthode non dispersive de filtration large bande.

\section{RÉSULTATS (Fig. 2)}

Les tirs en configuration jet d'Al seul $(\mathrm{a}, \mathrm{c}, \mathrm{e})$ montrent un plasma fortement inhomogène à la stagnation, avec un rapport de compression sur le rayon de l'ordre de 5 (diamètre de la source voisin de $4 \mathrm{~mm}$ ). Les signaux X présentent une impulsion principale, d'environ 10 à $15 \mathrm{~ns}$ de largeur, suivie d'une seconde impulsion 2 à 3 fois moins intense, 20 ns plus tard. L'énergie rayonnée dans la couche $K$ est voisine 
de $350 \mathrm{~J}$. La forte variation observée d'un tir à l'autre (facteur 7) peut s'expliquer par des conditions initiales faiblement reproductibles.

Les tirs en configuration jet d'Al sur fil central en $\mathrm{Al}$ (de $20 \mu \mathrm{m}$ à $50 \mu \mathrm{m}$ de diamètre) présentent deux impulsions de même intensité, également distantes de $20 \mathrm{~ns}$ (b,d,f). L'énergie rayonnée dans la couche $\mathrm{K}$ et le rapport de compression sont doublés, par rapport au cas liner seul. L'imagerie X confirme la stabilité, la reproductibilité et l'homogénéité de la colonne émissive observée dans les tirs avec un fil central en Al.

L'analyse d'un tir en configuration liner $\mathrm{Al}$ sur fil $\mathrm{Al}$ (diamètre égal à $50 \mu \mathrm{m}$ ) à l'aide d'un modèle collisionnel-radiatif hors équilibre thermodynamique local [4], a conduit à une interprétation, en bon accord avec l'ensemble des diagnostics : le plasma est composé d'un cœur froid et dense ( $350 \mathrm{eV}$, $\left.5.10^{20} \mathrm{~cm}^{-3}\right)$ entouré d'une couronne chaude et peu dense $\left(850 \mathrm{eV}, 10^{19} \mathrm{~cm}^{-3}\right)$.

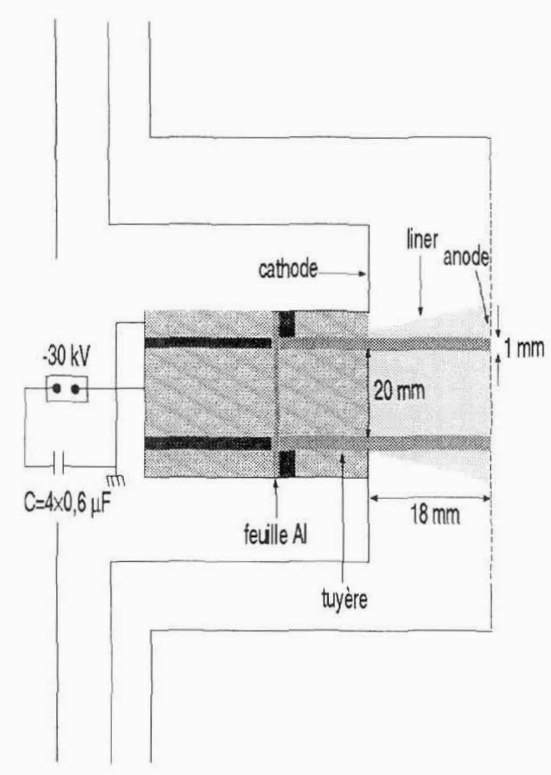

Figure 1 : Schéma de la diode AMBIORIX
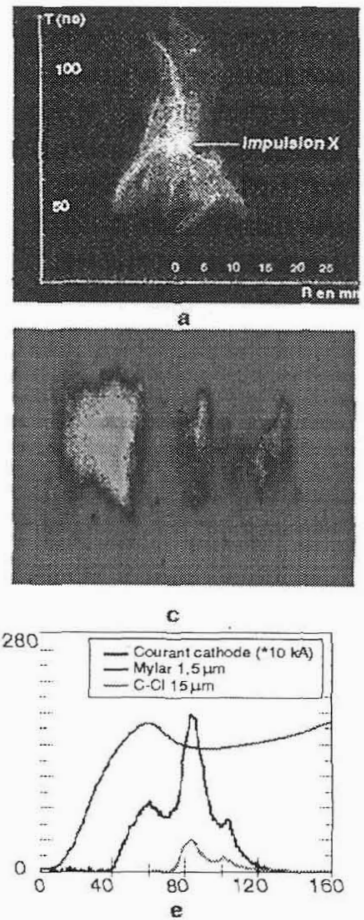

Figure 2 : Résultats expérimentaux de la caméra à balayage de fente $(a, b)$, de l'imagerie $X(c, d)$ et des signaux résolus en temps $(e, f)$.

\section{Références}

1. F.J. Wessel, Phys. Rev. L. 69 (22), 3181 (1992)

2. N.R. Pereira, J. Davis, J. Appl. Phys. 64 (3), (1988)

3. J.N. Barnier, Thèse de Doctorat de l'Université Bordeaux I, 1998

4. R.W. Lee, B.L. Whitten, J. of Quant. Spect. Radiat. Transfer, 32(1), 91 (1984) 